\title{
Wirksame Phytopharmatherapie bei Zystitis
}

— Für die Behandlung einer Blasenentzündung steht eine Reihe von Substanzklassen zur Verfügung. Speziell bei unkomplizierten Harnwegsinfektionen werden nicht selten vorschnell Antibiotika eingesetzt, die im weiteren Verlauf zu bakteriellen Resistenzen führen können.

Viele Experten empfehlen, bei unkomplizierten Blasenentzündungen pflanzliche Präparate einzunehmen. Die Wirkung der Bärentraube ist bereits seit dem 13. Jahrhundert bekannt. Trockenextrakt aus Beerentraubenblättern (Cystinol akut Dragees) besitzt eine antibakterielle sowie antientzündliche Wirkung, zudem wird die Adhäsion von Keimen an der Blasenschleimhaut gehemmt.

Der Wirkstoff Arbutin wird im Körper zu einer Hydrochinon-Verbindung umgewandelt, die mit dem Urin ausgeschieden und dabei von Bakterien im Harntrakt aufgenommen wird. In den Bakterien kommt es zur Spaltung der Verbindung und damit zur Freisetzung des freien Hydrochinons als bakterizider Substanz.

Die Ausscheidung der Substanz erfolgt unabhängig vom pH-Wert. Damit ist eine Ernährungsumstellung zur Alkalisierung des Harns während der Einnahme des Präparats nicht notwendig.

Die vielfach diskutierte Wirkung von Cranberries als Prävention gegen Zystitiden ist nach einer aktuellen Cochrane-Analyse nicht belegt. In einer Arbeit wurden Daten von 4.473 Studienteilnehmern ausgewertet. Das Trinken von Cranberry-Saft oder die Einnahme von Cranberry-Präparaten hatte keine signifikant präventive Wirkung [Jepson RG et al. Cochrane Database of Systematik Reviews 2012, Issue 10, CD001321].

Nach Informationen von Schaper \& Brümmer, Salzgitter

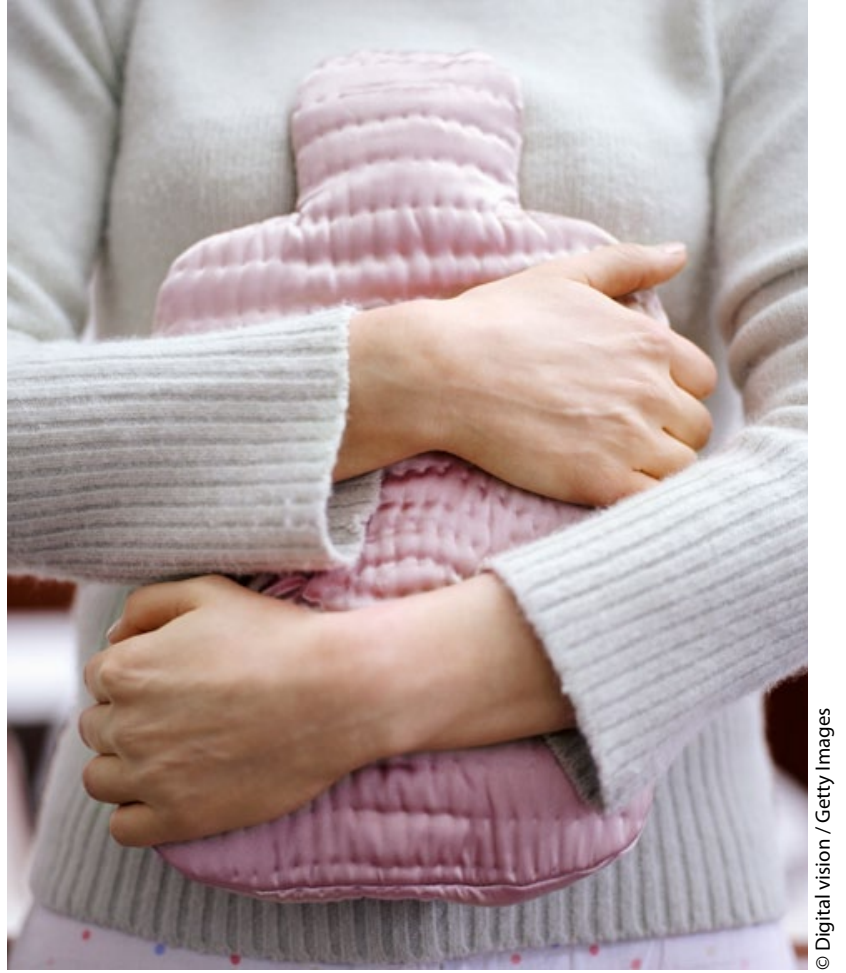

Unkomplizierte Zystitis - kein vorschneller Griff zu Antibiotika!

\section{Risiko für febrile Neutropenien beachten}

_ Beim metastasierten kastrationsresistenten Prostatakarzinom (mCRPC) sind dank neuer Therapiestrategien wie der sequenziellen Chemotherapie mit Docetaxel und Cabazitaxel heute lange Überlebenszeiten möglich. Allerdings sind diese Behandlungen mit Nebenwirkungen für die Patienten behaftet. Besondere Aufmerksamkeit muss der Vermeidung einer febrilen Neutropenie gelten. Diese kann im praktischen Alltag durch den prophylaktischen Einsatz von Granulozyten-koloniestimulierenden Faktoren (G-CSF) verhindert werden. Dr. Joachim Kleeberg, Stuttgart, machte beim Kongress der Deutschen Gesellschaft für Urologie 2012 in Leipzig darauf aufmerksam, dass febrile Neutropenien im Rahmen zunehmend eingesetzter Chemotherapeutika bei niedergelassenen Urologen mehr Beachtung erfahren sollten. Als besonders bewährt zur Primärprophylaxe nannte er den nur einmal pro Zyklus zu verabreichenden pegylierten G-CSF Pegfilgrastim
(Neulasta ${ }^{\oplus}$ ). Die Einmalgabe pro Chemotherapiezyklus bietet besonders den ambulant behandelten Prostatakarzinompatienten deutliche Vorteile.

„Im Compassionate Use Programm (CUP) wurde von Anfang an ein proaktives Nebenwirkungsmanagement mit entsprechendem G-CSF-Support gefahren, sodass die Rate an Nebenwirkungen wesentlich reduziert werden konnte", erläuterte Kleeberg. Die Sorge, dass Patienten die Behandlung mit Cabazitaxel nicht ausreichend gut vertragen, sei bei entsprechender Aufklärung und regelmäBigem Monitoring unbegründet.

Dazu gehöre aber auch zwingend der präventive G-CSF-Einsatz bei gefährdeten Patienten. Bei einer Chemotherapie mit einem moderaten Risiko von 10-20\% empfehlen die Leitlinien der European Organisation for Research and Treatment of Cancer (EORTC), patientenbezogene Faktoren wie das Lebensalter mit zu berücksichtigen, um das individuelle Gesamtrisiko beurteilen zu kön- nen. So ist bei über 65-jährigen Patienten, aber auch bei fortgeschrittener Erkrankung und/oder vorliegenden Komorbiditäten die Gefahr größer, dass sie im Verlauf der Behandlung eine febrile Neutropenie entwickeln.

Unter den verschiedenen Wachstumsfaktoren hob der Urologe Pegfilgrastim hervor: „Dieses bietet einen besseren Schutz und eventuell auch eine bessere Wirkung als Filgrastim. Dazu kommt noch der Vorteil, dass die Dosierungsschemata und Therapietage nicht variieren", erklärte Kleeberg. Um den Einsatz von Pegfilgrastim richtig zu gestalten, müsse nur einmal pro Zyklus und zwar 24 Stunden nach der letzten Chemotherapie-Dosis $6 \mathrm{mg}$ subkutan verabreicht werden.

Nach Informationen von Amgen, München 